\title{
Analysis of Some Difference Approximations for a Singular Perturbation Problem Without Turning Points
}

\author{
By R. Bruce Kellogg* and Alice Tsan
}

\begin{abstract}
Some three point difference schemes are considered for a singular perturbation problem without turning points. Bounds for the discretization error are obtained which are uniformly valid for all $h$ and $\epsilon>0$. The degeneration of the order of the schemes at $\epsilon=0$ is considered.
\end{abstract}

1. Introduction. We consider the two point boundary value problem

$$
\begin{gathered}
L y \equiv-\epsilon y^{\prime \prime}+p y^{\prime}+q y=f, \quad y(0)=\alpha, \quad y(1)=\beta, \\
p(x)>a>0, \quad q(x) \geqslant 0,
\end{gathered}
$$

where $\epsilon>0$ is a small parameter. It is well known that the solution $y(x, \epsilon)$ of this problem converges, as $\epsilon \rightarrow 0$, and for $0 \leqslant x<1$, to the solution $v(x)$ of the reduced problem

$$
p v^{\prime}+q v=f, \quad v(0)=\alpha .
$$

The loss of a boundary condition at $x=1$ in the reduced problem results in a "boundary layer" in the solution $y$, for small $\epsilon$. It is also well known [1, p. 300] that a reasonable difference approximation to (1) may give inaccurate results for small $\epsilon$. In particular, the usual centered three point $O\left(h^{2}\right)$ difference approximation has this property. In this paper we analyse three difference operators, $L_{h}^{k}, k=1,2$, 3 , on a uniform mesh of size $h$, for use in the approximate solution of (1.1). Each of the operators results in a tridiagonal, diagonally dominant matrix with negative offdiagonal entries. The operator $L_{h}^{1}$, which has been frequently proposed for such problems, uses a one sided difference approximation to the first derivative, and gives an $O(h)$ approximation to (1.1). The operators $L_{h}^{2}$ and $L_{h}^{3}$ give $O\left(h^{2}\right)$ approximations to (1.1). $L_{h}^{3}$ was proposed independently by $I^{\prime}$ in [2] and by K. E. Barrett and others [3]. The operator $L_{h}^{2}$ was considered by Samarskii (see [9]). Each of the three approximate schemes behaves reasonably for $\epsilon$ small, and upon setting $\epsilon=0$ in $L_{h}^{2}$ and $L_{h}^{3}$, an $O(h)$ approximation is obtained for the reduced problem (1.2).

Our purpose is to give bounds for the discretization error for the three schemes that are uniform in $\epsilon$ and $h$. Our error bounds contain a term that gives the effect of the boundary layer, and the bounds demonstrate that the boundary layer does not

Received February 22, 1978.

AMS (MOS) subject classifications (1970). Primary 65L10, 34E1 5.

*Work supported in part by the National Institutes of Health under contract \#HI52900. Copyright $\odot$ 1978. American Mathematical Society 
"pollute" the error away from $x=1$. In the case of the second order schemes, our bounds contain a term of the form $h^{2} /(h+\epsilon)$, reflecting the loss of one order of accuracy in the error as $\epsilon \longrightarrow 0$. We also show that, among a certain class of difference schemes, this loss of an order of accuracy as $\epsilon \rightarrow 0$ is unavoidable. Finally, we give some numerical results illustrating our bounds. To obtain our error bounds we utilize the positivity of the difference schemes and a comparison function that is designed to handle the effect of the boundary layer in the truncation error. This technique may be of use in other problems.

The literature on the numerical solution of singular perturbation problems is large. A useful discussion of a variety of problems is contained in Dorr [1]. Il'in [2] gives an $O(h)$ error bound for his scheme that is uniform in $\epsilon$. We give a different proof of this result. Abrahamsson, Keller, and Kreiss [4] give an asymptotic expansion of the difference solution in $h$ and $\epsilon$. Some other methods for the numerical solution of singular perturbation problems are given, e.g., in [5].

In Section 2 we given some properties of solutions of (1.1), and in Section 3 we state the difference approximations that are being studied. The main results are contained in Section 4, and some numerical examples are presented in Section 5. Throughout the paper we let $c, c_{1}, \ldots$ denote positive constants that may take different values in different formulas, but that are always independent of $h$ and $\epsilon$. We assume that the parameter $\epsilon$ satisfies $0<\epsilon \leqslant 1$. We assume that the functions $p(x)$, $q(x)$, and $f(x)$ are sufficiently differentiable for our purposes, but we shall not write out the assumptions in each instance.

2. Differentiability Properties of the Solution. To estimate the error in our difference approximations we shall require a bound for the derivatives of the solution of (1.1) that is valid for all $\epsilon \in(0,1]$. To analyse the $\mathrm{Il}^{\prime}$ in scheme we require more precise information on the behavior of the solution. These results are contained in Lemmas 2.3 and 2.4. To obtain the results, we require some information about the solutions of

$$
L y=g(x, \epsilon), \quad y(0)=\alpha, \quad y(1)=\beta,
$$

where $g$ satisfies

$$
\left|g^{(i)}(x, \epsilon)\right| \leqslant K\left(1+\epsilon^{-i-1} \exp \left(-a \epsilon^{-1}(1-x)\right)\right) .
$$

We will say that $g$ is of class $(K, j)$ if $(2.2)$ holds for $0 \leqslant i \leqslant j$. Our first result is

LEMMA 2.1. The problem $(2.1)$ has a unique solution $y$. If $g$ is of class $(K, 0)$, then $|y(x)| \leqslant c$ where $c$ depends only on $\alpha, \beta$, and $K$.

Proof. The existence and uniqueness of a solution follows easily from the maximum principle [6]. A computation shows that

$$
L(1+x) \geqslant c_{1}, \quad L\left(\exp \left(-a \epsilon^{-1}(1-x)\right)\right) \geqslant c_{2} \epsilon^{-1} \exp \left(-a \epsilon^{-1}(1-x)\right) .
$$

Hence we may choose $c_{3}$ and $c_{4}$ so that

$$
z(x)=c_{3} e^{-x}+c_{4} \exp \left(-a \epsilon^{-1}(1-x)\right)
$$


satisfies $L(z \pm y) \geqslant 0, z(0) \geqslant|\alpha|, z(1) \geqslant|\beta|$. From the maximum principle, $|y(x)|$ $\leqslant z(x) \leqslant c$.

Lemma 2.2. Let $g$ be of class $(K, j)$. Then the solution $y$ of $(2.1)$ satisfies $\left|y^{(i)}(1)\right| \leqslant c \epsilon^{-i}, 1 \leqslant i \leqslant j+2$, where $c>0$ does not depend on $\epsilon$ -

Proof. From (2.1), $-\epsilon y^{\prime \prime}+p y^{\prime}=h$ where $h=g-q y$. Let $P(x)$ be an indefinite integral of $p$. Then we obtain

$$
y(x)=y_{p}(x)+K_{1}+K_{2} \int_{x}^{1} \exp \left(-\epsilon^{-1}(P(1)-P(t))\right) d t,
$$

where

$$
y_{p}(x)=-\int_{x}^{1} z(t) d t, \quad z(x)=\int_{x}^{1} \epsilon^{-1} h(t) \exp \left(-\epsilon^{-1}(P(t)-P(x))\right) d t .
$$

Using the inequality

$$
\exp \left(-\epsilon^{-1}(P(t)-P(x))\right) \leqslant \exp \left(-a \epsilon^{-1}(t-x)\right), \quad x \leqslant t,
$$

and (2.2),

$$
\begin{aligned}
|z(x)| & \leqslant c \epsilon^{-1} \int_{x}^{1}\left[\exp \left(-\epsilon^{-1} a(t-x)\right)+K \epsilon^{-1} \exp \left(-\epsilon^{-1} a(1-x)\right)\right] d t \\
& \leqslant c\left[1+\epsilon^{-2}(1-x) \exp \left(-\epsilon^{-1} a(1-x)\right)\right] .
\end{aligned}
$$

Hence $\left|y_{p}(x)\right| \leqslant c$. The constants $K_{1}$ and $K_{2}$ must satisfy

$$
K_{1}+K_{2} \int_{0}^{1} \exp \left\{-\epsilon^{-1}(P(1)-P(t))\right\} d t=\alpha-y_{p}(0), \quad K_{1}=\beta .
$$

Since $p(x)$ is bounded on $(0,1), P(1)-P(t) \leqslant c(1-t)$. Hence

$$
\int_{0}^{1} \exp \left\{\left(-\epsilon^{-1}(P(1)-P(t))\right\} d t \geqslant c \epsilon,\right.
$$

and we find that $K_{2} \leqslant c \epsilon^{-1}$. Hence, $\left|y^{\prime}(1)\right|=\left|K_{2}\right| \leqslant c \epsilon^{-1}$, so the inequality is proved with $i=1$. If $i>1$, the result is obtained by induction and repeated differentiations of (2.1).

Lemma 2.3. Let $g$ be of class $(K, j)$. Then the solution $y$ of $(2.1)$ satisfies

$$
\left|y^{(i)}(x)\right| \leqslant c\left\{1+\epsilon^{-i} \exp \left(-a \epsilon^{-1}(1-x)\right)\right\}, \quad 0 \leqslant i \leqslant j+1,
$$

where $c>0$ does not depend on $\epsilon$.

Proof. The proof is by induction. From Lemma 2.1, the inequality holds for $i=0$. Differentiating both sides of (2.1) $i-1$ times and setting $z=y^{(i)}$, we have $-\epsilon z^{\prime}+p z=h$, where $h$ depends on $y, p, q, g$, and their derivatives of order up to and including $i-1$. Using (2.2) and the inductive hypothesis,

$$
h(x) \leqslant c\left\{1+\epsilon^{-i} \exp \left(-a \epsilon^{-1}(1-x)\right)\right\} .
$$

Let $P$ be an indefinite integral of $p$. Then

$$
\begin{aligned}
z(x)= & z(1) \exp \left(-\epsilon^{-1}(P(1)-P(x))\right) \\
& +\epsilon^{-1} \int_{x}^{1} h(t) \exp \left(-\epsilon^{-1}(P(t)-P(x))\right) d t .
\end{aligned}
$$

From (2.4), (2.6), and Lemma 2.2, 


$$
\begin{aligned}
|z(x)| \leqslant & c \epsilon^{-i} \exp \left(-a \epsilon^{-1}(1-x)\right) \\
& +c \epsilon^{-1} \int_{x}^{1}\left\{\exp \left(-a \epsilon^{-1}(t-x)\right)+\epsilon^{-i} \exp \left(-a \epsilon^{-1}(1-x)\right)\right\} d t,
\end{aligned}
$$

and the desired inequality follows from this.

Remark 1. In particular, (2.5) holds when $y$ is the solution of (1.1). This is used in the analysis of the difference schemes $L_{h}^{k}, k=1,2$.

Lemma 2.4. Let $y$ satisfy (1.1). Then

$$
y(x)=\gamma \exp \left(-p(1) \epsilon^{-1}(1-x)\right)+z(x),
$$

where $|\gamma| \leqslant c_{1}$, and

$$
\left|z^{(i)}(x)\right| \leqslant c_{2}\left\{1+\epsilon^{-i+1} \exp \left(-a \epsilon^{-1}(1-x)\right)\right\},
$$

with $c_{1}>0$ and $c_{2}>0$ independent of $\epsilon$.

Proof. Set $v(x)=\exp \left(-p(1) \epsilon^{-1}(1-x)\right)$, and set $\gamma=\epsilon y^{\prime}(1) / p(1)$. Then from Lemma 2.2, we see that $|\gamma| \leqslant c_{1}$ where $c_{1}>0$ does not depend on $\epsilon$. Set $z(x)=$ $y(x)-\gamma v(x)$. Then $z^{\prime}(1)=0$. Differentiating both sides of (2.3) and setting $x=0$, we find that $\left|y^{\prime}(0)\right| \leqslant c$. Calculating

$$
L z=f-q y+\gamma[p(1)-p(x)] v^{\prime}+q z=g
$$

and differentiating once, we get $L z^{\prime}=g^{\prime}-p^{\prime} z^{\prime}-q^{\prime} z$. Using (2.5), we see that $y^{\prime}$ is of class $(K, j)$. Hence, $z^{\prime}=y^{\prime}-\gamma v^{\prime}$ is of class $(K, j)$, and we find that the function $L z^{\prime}$ is of class $(K, j)$. Using Lemma 2.3 with $y$ replaced by $z^{\prime}$, we find that $z$ satisfies (2.7), and the lemma is proved.

Remark 2. Lemma 2.4 is used in the analysis of the Il'in scheme, $L_{h}^{3}$.

3. The Difference Equations. Let $(0,1)$ be divided into $N$ uniformly spaced mesh intervals, with mesh spacing $h=N^{-1}$ and with mesh points $x_{i}=i h, 0 \leqslant i \leqslant$ $N$. Using the usual notations for divided differences,

$$
\begin{aligned}
D_{+} u_{i} & =\left(u_{i+1}-u_{i}\right) / h, & D_{-} u_{i} & =\left(u_{i}-u_{i-1}\right) / h, \\
D_{0} u_{i} & =\left(u_{i+1}-u_{i-1}\right) / 2 h, & D_{+} D_{-} u_{i} & =\left(u_{i+1}-2 u_{i}+u_{i-1}\right) / h^{2},
\end{aligned}
$$

we define our difference operators by

$$
\begin{aligned}
L_{h}^{1} u_{i} & =-\epsilon D_{+} D_{-} u_{i}+p_{i} D_{-} u_{i}+q_{i} u_{i}, \\
L_{h}^{2} u_{i} & =\frac{-\epsilon}{1+h p_{i} / 2 \epsilon} D_{+} D_{-} u_{i}+p_{i} D_{-} u_{i}+q_{i} u_{i}, \\
L_{h}^{3} u_{i} & =-\frac{1}{2} p_{i} h\left(\operatorname{coth} \frac{p_{i} h}{2 \epsilon}\right) D_{+} D_{-} u_{i}+p_{i} D_{0} u_{i}+q_{i} u_{i},
\end{aligned}
$$

where $p_{i}=p\left(x_{i}\right), q_{i}=q\left(x_{i}\right)$. We shall also write $f_{i}=f\left(x_{i}\right)$.

In this section we give some elementary facts concerning the positivity and the truncation errors of these operators.

Lemma 3.1. For $k=1,2,3$, the system $L_{h}^{k} u_{i}=f_{i}, 1 \leqslant i \leqslant N-1$, with $u_{0}$ and $u_{N}$ specified, has a solution. If $L_{h}^{k} u_{i} \leqslant L_{h}^{k} v_{i}, 1 \leqslant i \leqslant N-1$, and if $u_{0} \leqslant v_{0}, u_{N}$ $\leqslant v_{N}$, then $u_{i} \leqslant v_{i}, 1 \leqslant i \leqslant N-1$. 
Proof. The equations $L_{h}^{k} u_{i}=f_{i}, 1 \leqslant i \leqslant N-1$, may be regarded as a system of $N-1$ linear equations in the unknowns $u_{i}, 1 \leqslant i \leqslant N-1$, where for $i=1$ and $i=N-1$, the terms involving $u_{0}$ and $u_{N}$ have been moved to the right-hand side. It is easy to see that the matrix of coefficients is diagonally dominant and has nonpositive off diagonal entries. Hence, the matrix is an irreducible $M$ matrix [7], and so has a positive inverse. Hence, the solution $u_{i}, 1 \leqslant i \leqslant N-1$, exists and, if the $v_{i}$ are as described in the lemma, $u_{i} \leqslant v_{i}, 1 \leqslant i \leqslant N-1$.

The following lemma, whose proof is a computation, enables one to give a bound, that is uniform in $\epsilon$ and $h$, for the norm of the inverse of $L_{h}^{k}$.

Lemma 3.2. Let $z_{i}=\left(1+x_{i}\right), 0 \leqslant i \leqslant N$. Then $L_{h}^{k} z_{i} \leqslant c, k=1,2,3$, where $c>0$ does not depend on $\epsilon$.

We now consider the truncation error associated with the operators $L_{h}^{k}$. If $y(x)$ is a smooth function, we define $\tau_{i}^{k}=L_{h}^{k} y_{i}-L y\left(x_{i}\right)$. We require estimates for $\tau_{i}^{k}$ that are in integrable form.

LEMma 3.3. There is a constant $c>0$ depending only on $p(x)$ such that

$$
\begin{aligned}
& \left|\tau_{i}^{k}\right| \leqslant c \int_{x_{i-1}}^{x_{i+1}}\left[\epsilon\left|y^{(3)}(t)\right|+\left|y^{(2)}(t)\right|\right] d t, \quad k=1,3, \\
& \left|\tau_{i}^{2}\right| \leqslant \frac{c h}{h+\epsilon} \int_{x_{i-1}}^{x_{i+1}}\left[\epsilon^{2}\left|y^{(4)}(t)\right|+\epsilon\left|y^{(3)}(t)\right|+\left|y^{(2)}(t)\right|\right] d t, \\
& \left|\tau_{i}^{3}\right| \leqslant \operatorname{ch} \int_{x_{i-1}}^{x_{i+1}}\left[\epsilon\left|y^{(4)}(t)\right|+\left|y^{(3)}(t)\right|+(h+\epsilon)^{-1}\left|y^{(2)}(t)\right| d t .\right.
\end{aligned}
$$

Proof. By repeated use of the fundamental theorem of calculus, or by Peano's theorem [8, p. 70] we obtain the formulas

$$
\begin{aligned}
D_{-} y(x)-y^{(1)}(x)= & -h^{-1} \int_{x-h}^{x}(s-x+h) y^{(2)}(s) d s \\
=-\frac{1}{2} h y^{(2)}(x)+\frac{1}{2} h^{-1} \int_{x-h}^{x}(s+h-x)^{2} y^{(3)}(s) d s, & -\frac{1}{2} h^{-1} \int_{x-h}^{x}(s-x+h) y^{(2)}(s) d s \\
D_{0} y(x)-y^{(1)}(x)= & \frac{1}{2} h^{-1} \int_{x}^{x+h}(x+h-s) y^{(2)}(s) d s \\
= & \frac{1}{4} h^{-1} \int_{x-h}^{x}(s+h-x)^{2} y^{(3)}(s) d s \\
& +\frac{1}{4} h^{-1} \int_{x}^{x+h}(x+h-s)^{2} y^{(3)}(s) d s \\
D_{+} D_{-} y(x)-y^{2}(x)= & \frac{-1}{2} h^{-2} \int_{x-h}^{x}(s+h-x)^{2} y^{(3)}(s) d s \\
& +\frac{1}{2} h^{-2} \int_{x}^{x+h}(x+h-s)^{2} y^{(3)}(s) d s \\
= & \frac{1}{6} h^{-2} \int_{x-h}^{x}(s+h-x)^{3} y^{(4)}(s) d s \\
& +\frac{1}{6} h^{-2} \int_{x}^{x+h}(x+h-s)^{3} y^{(4)}(s) d s .
\end{aligned}
$$


Let us designate $R_{m, n}$ to be a quantity which satisfies

$$
\left|R_{m, n}\right| \leqslant c h^{m} \int_{x_{i-1}}^{x_{i+1}}\left|y^{(n)}(s)\right| d s .
$$

Using (3.4) and (3.6) and the formula

$$
L_{h}^{1} y\left(x_{i}\right)-L y\left(x_{i}\right)=-\epsilon\left[D_{+} D_{-} y\left(x_{i}\right)-y^{(2)}\left(x_{i}\right)\right]+p_{i}\left[D_{-} y\left(x_{i}\right)-y^{(1)}\left(x_{i}\right)\right],
$$

we obtain

$$
\tau_{i}^{1}=\epsilon R_{0,3}+R_{0,2} \text {. }
$$

This proves (3.1) with $k=1$. Next, using (3.7) with the higher order error estimates of (3.4) and (3.6), we get

$$
L_{h}^{1} y\left(x_{i}\right)-L y\left(x_{i}\right)=\epsilon R_{1,4}-1 / 2 p_{i} h y^{(2)}\left(x_{i}\right)+R_{1,3} .
$$

Using the differential equation to eliminate $y^{(2)}\left(x_{i}\right)$, and using (3.4) again, we obtain

$$
y^{(2)}\left(x_{i}\right)=\epsilon^{-1}\left[p_{i} D_{-} y\left(x_{i}\right)+q_{i} y\left(x_{i}\right)-f_{i}\right]+\epsilon^{-1} R_{0,2},
$$

so

$$
\begin{gathered}
-\epsilon D_{+} D_{\_} y\left(x_{i}\right)+\left(1+p_{i} h / 2 \epsilon\right)\left\{p_{i} D_{\beth} y\left(x_{i}\right)+q_{i} y\left(x_{i}\right)-L y\left(x_{i}\right)\right\} \\
=\epsilon R_{1,4}+R_{1,3}+\epsilon^{-1} h R_{0,2} .
\end{gathered}
$$

Dividing both sides by $\left(1+p_{i} h / 2 \epsilon\right)$, we see that the left side gives $\tau_{i}^{2}$, and the right side gives the bound in (3.2). To analyse $\tau_{i}^{3}$, we start with the formula

$$
\begin{aligned}
\tau_{i}^{3}= & -\epsilon\left[\frac{p_{i} h}{2 \epsilon} \operatorname{coth} \frac{p_{i} h}{2 \epsilon}-1\right] D_{+} D_{-} y\left(x_{i}\right)-\epsilon\left[D_{+} D_{-} y\left(x_{i}\right)-y^{(2)}\left(x_{i}\right)\right] \\
& +p_{i}\left[D_{0} y\left(x_{i}\right)-y^{(1)}\left(x_{i}\right)\right] .
\end{aligned}
$$

Since $g(t)=t$ coth $t$ satisfies $g(0)=1, g(t)=g(-t)$, we have $|g(t)-1| \leqslant c t^{2}$ for $t \leqslant 1$. Since coth $t \rightarrow 1$ as $t \rightarrow \infty,|g(t)-1| \leqslant c t$ for $t \geqslant 1$. Hence

$$
|t \operatorname{coth} t-1| \leqslant c t^{2} /(1+t), \quad t \geqslant 0 \text {. }
$$

It is easily seen that

$$
\left|D_{+} D_{-} y\left(x_{i}\right)\right| \leqslant h^{-1} \int_{x_{i-1}}^{x_{i+1}}\left|y^{(2)}(s)\right| d s .
$$

Using these inequalities to estimate the first term of (3.8), and using (3.5) and (3.6) to estimate the two remaining terms, we easily obtain (3.1) with $k=3$ and (3.3), completing the proof.

4. Error Bounds. In this section we derive error bounds for our difference schemes $L_{h}^{k}, k=1,2,3$. We set $r_{1}=1+a h \epsilon^{-1}, r_{2}=r_{1}+1 / 2 a^{2} h^{2} \epsilon^{-2}, r_{3}=$ $\exp \left(a h \epsilon^{-1}\right)$. We first require some inequalities.

LEMMA 4.1. (a) $\left(h^{k} / \epsilon^{k}\right) r_{3}^{-(N-i)} \leqslant c r_{2}^{-(N-i)} \leqslant c r_{1}^{-(N-i)}, 0 \leqslant i<N$, or, $0 \leqslant i$ $\leqslant N$ if $k=0$, where $k$ is a nonnegative integer and $c$ depends only on $k$;

(b) $r_{2}^{-(N-i)} \leqslant r_{1}^{-(N-i)} \leqslant \exp \left\{-a\left(1-x_{i}\right) /(a h+\epsilon)\right\}$;

(c) $r_{2}^{-(N-i)} \leqslant r_{1}^{-(N-i)} \leqslant c \exp \left\{-\epsilon^{-1} \bar{a}\left(1-x_{i}\right)\right\}$, where $h \leqslant \epsilon$, and $\bar{a} \in(0, a)$ is a constant depending only on $a$. 
Proof. (a) Since $e^{t} \geqslant 1+t+t^{2} / 2$ and $t^{k}\left(1+t+t^{2} / 2\right) e^{-t} \leqslant c$, for all $t \geqslant 0$ where $c$ is some constant, these imply that $t \geqslant \ln \left(1+t+t^{2} / 2\right)$ and $k \ln t \leqslant t-$ $\ln \left(1+t+t^{2} / 2\right)+\ln c$. It follows that

$$
k \ln t \leqslant \frac{1-x_{i}}{h}\left[t-\ln \left(1+t+\frac{t^{2}}{2}\right)\right]+\ln c .
$$

Let $t=a h / \epsilon$, then

$$
\ln \frac{a^{k} h^{k}}{\epsilon^{k}}-\frac{1-x_{i}}{h} \cdot \frac{a h}{\epsilon} \leqslant-\frac{1-x_{i}}{h} \ln \left(1+\frac{a h}{\epsilon}+\frac{a^{2} h^{2}}{2 \epsilon^{2}}\right)+\ln c .
$$

Taking the exponential of both sides, we get the first inequality. The second inequality is easy to prove.

(b) We only prove the second inequality. We have

$$
\begin{gathered}
B=\left(1+\frac{a h}{\epsilon}\right)^{-\left(1-x_{i}\right) / h}=\left(1-\frac{a h}{a h+\epsilon}\right)^{\left(1-x_{i}\right) / h}, \\
\ln B=\frac{1-x_{i}}{h} \ln \left(1-\frac{a h}{a h+\epsilon}\right)<\frac{1-x_{i}}{h}\left[-\frac{a h}{a h+\epsilon}\right]=-\frac{\left(1-x_{i}\right) a}{a h+\epsilon} .
\end{gathered}
$$

Taking the exponential of both sides, we get the results.

(c) To improve the upper bound in (b) when $h \leqslant \epsilon$, we start with the inequality

$$
\exp (a t) \leqslant 1+a t, \quad 0 \leqslant t \leqslant 1,
$$

where $\bar{a} \in(0, a)$ depends only on $a$. Setting $t=h / \epsilon$, we obtain $r_{1}^{-1} \leqslant \exp \left(-\bar{a} h \epsilon^{-1}\right)$, and raising both sides to the power $N-i$, we get the result.

The next lemma will be used, with Lemmas 3.2 and 3.1, to convert bounds for the truncation error into bounds for the discretization error.

LEMMA 4.2. There is a $c>0$ depending only on $p(x)$ and a such that, for $k=$ $1,2,3$,

$$
L_{h}^{k} r_{k}^{-(N-i)} \geqslant \frac{c}{\max (h, \epsilon)} r_{k}^{-(N-i)}
$$

Proof. A computation shows that

$$
L_{h}^{1} r_{1}^{i} \geqslant h^{-1}\left(r_{1}-1\right)\left(p\left(x_{i}\right)-a\right) r_{1}^{i-1} .
$$

If $h \geqslant \epsilon$, then there is a constant $c_{1}$ such that $r_{1}-1 \geqslant c_{1} r_{1}$, so $L_{h}^{1} r_{1}^{i} \geqslant c_{2} h^{-1} r_{1}^{i}$, and we obtain (4.2) in this case. If $h \leqslant \epsilon$, since $r_{1}-1=a h \epsilon^{-1}$ we have

$$
L_{h}^{1} r_{1}^{i} \geqslant c_{3} \epsilon^{-1} r_{1}^{i-1}=c_{3} r_{1}^{i} /(\epsilon+a h) \geqslant c_{4} \epsilon^{-1} r_{1}^{i},
$$

and we obtain (4.2) in this case. A similar argument is used when $k=2$. For $k=$ 3 , a computation gives

$$
L_{h}^{3} r_{3}^{i} \geqslant\left(p_{i} / 2 h r_{3}\right)\left(r_{3}-1\right)^{2} A r_{3}^{i},
$$


where

$A=\frac{r_{3}+1}{r_{3}-1}-\operatorname{coth} \frac{p_{i} h}{2 \epsilon}=\operatorname{coth} \frac{a h}{2 \epsilon}-\operatorname{coth} \frac{p_{i} h}{2 \epsilon}=\sinh \frac{\left(p_{i}-a\right) h}{2 \epsilon} / \sinh \frac{a h}{2 \epsilon} \sinh \frac{p_{i} h}{2 \epsilon}$.

If $h \leqslant \epsilon$, since $c_{1} t \leqslant \sinh t \leqslant c_{2} t$ for $0 \leqslant t \leqslant c$, we have $A \geqslant c \epsilon / h$. Since, in this case,

$$
\left(r_{3}-1\right)^{2} r_{3}^{-1}=4 \sinh ^{2} \frac{a h}{2 \epsilon} \geqslant c \frac{h^{2}}{\epsilon^{2}}
$$

we have

$$
L_{h}^{3} r_{3}^{i} \geqslant c r_{3}^{i} / \epsilon
$$

If $h \geqslant \epsilon$, since $c_{1} e^{t} \leqslant \sinh t \leqslant c_{2} e^{t}$ for $c \leqslant t<\infty$, we have $A \geqslant c e^{-a h / \epsilon}=c r_{3}^{-1}$. Since, in this case, $r_{3}-1 \geqslant c r_{3}$, we have

$$
L_{h}^{3} r_{3}^{i} \geqslant c r_{3}^{i} / \epsilon
$$

and the proof is complete.

Remark. The qualities $r_{k}$ arise in the following way. If, in the definition of $L_{h}^{k}$, we set $q(x) \equiv 0, p(x) \equiv a$, then $L_{h}^{k} r_{k}^{i}=0, k=1,2,3$. We also note that $r_{k}^{-1}$, $k=1,2$, is the Padé approximation of type $(0, k)$ to $r_{3}^{-1}=\exp \left(-a h \epsilon^{-1}\right)$.

We now prove the main theorems of the paper. Let $y(x)$ be the solution of (1.1), and let $y=\gamma v+z$ be the decomposition of Lemma 2.4, where we have set $v(x)=\exp \left(-p(1) \epsilon^{-1}(1-x)\right)$. We let $y_{h i}^{k}$ be the solution of the system $L_{h}^{k} y_{h i}^{k}=$ $L y\left(x_{i}\right), 1 \leqslant i \leqslant N-1, y_{h 0}^{k}=y(0), y_{h N}^{k}=y(1)$. We define the mesh functions $v_{h i}^{k}$ and $z_{h i}^{k}$ in a similar manner. Our first result is

THEOREM 4.1. There is a $c>0$, independent of $h$ and $\epsilon$ such that

$$
\begin{array}{ll}
\left|y\left(x_{i}\right)-y_{h i}^{1}\right| \leqslant c h\left[1+\epsilon^{-1} \exp \left(-\bar{a} \epsilon^{-1}\left(1-x_{i}\right)\right)\right], & h \leqslant \epsilon, \\
\left|y\left(x_{i}\right)-y_{h i}^{1}\right| \leqslant c\left[h+\exp \left(-a\left(1-x_{i}\right) /(a h+\epsilon)\right)\right], & h \geqslant \epsilon,
\end{array}
$$

where $\bar{a}$ is as in Lemma 4.1.

Proof. We first suppose that $h \leqslant \epsilon$. We obtain from Lemma 3.3 and 2.3,

$$
\begin{aligned}
\left|\tau_{i}^{1}\right| & \leqslant c\left\{\epsilon^{-2} \int_{x_{i-1}}^{x_{i+1}} \exp \left(-\epsilon^{-1} a(1-t)\right) d t+h\right\} \\
& \leqslant c \epsilon^{-1} \sinh \left(a h \epsilon^{-1}\right) \exp \left(-\epsilon^{-1} a\left(1-x_{i}\right)\right)+c h .
\end{aligned}
$$

Since $\sinh t \leqslant c t$ for $t$ bounded, we obtain, using Lemma 4.1(a)

$$
\left|\tau_{i}^{1}\right| \leqslant \operatorname{ch}\left\{\epsilon^{-2} r_{3}^{-(N-i)}+1\right\} \leqslant \operatorname{ch}\left\{\epsilon^{-2} r_{1}^{-(N-i)}+1\right\} .
$$

Since $L_{h}^{1}\left(y\left(x_{i}\right)-y_{h i}^{1}\right)=\tau_{i}^{1}$, we may use Lemmas $3.2,4.2$, and 3.1 to obtain

$$
\left|y\left(x_{i}\right)-y_{h i}^{1}\right| \leqslant \operatorname{ch}\left\{\epsilon^{-1} r_{1}^{-(N-i)}+1\right\} .
$$

We obtain the desired inequality from Lemma 4.1(c). To treat the case $h \geqslant \epsilon$, we use the decomposition $y=\gamma v+z, y_{h i}^{1}=\gamma v_{h i}^{1}+z_{h i}^{1}$. We have 


$$
\left|y\left(x_{i}\right)-y_{h i}^{1}\right| \leqslant c\left\{\left|v\left(x_{i}\right)-v_{h i}^{1}\right|+\left|z\left(x_{i}\right)-z_{h i}^{1}\right|\right\} .
$$

To estimate the $z$ term, we use Lemmas 3.3 and 2.4 to obtain

$$
\begin{aligned}
\mid L_{h}^{1}\left(z\left(x_{i}\right)\right. & \left.-z_{h i}^{1}\right)|=| L_{h}^{1} z\left(x_{i}\right)-L z\left(x_{i}\right) \mid \\
& \leqslant c \int_{x_{i-1}}^{x_{i+1}}\left\{\epsilon\left|z^{(3)}(t)\right|+\left|z^{(2)}(t)\right|\right\} d t \\
& \leqslant c\left\{\epsilon^{-1} \int_{x_{i-1}}^{x_{i+1}} \exp \left(-\epsilon^{-1} a(1-t)\right) d t+h\right\} \\
& \leqslant c \sinh \left(a h \epsilon^{-1}\right) \exp \left(-\epsilon^{-1} a\left(1-x_{i}\right)\right)+c h
\end{aligned}
$$

Since $\sinh t \leqslant c e^{t}$ for $t \geqslant c_{1}$, we have, using Lemma 4.1(a),

$$
\left.\mid L_{h}^{1}\left(z\left(x_{i}\right)-z_{h i}^{1}\right)\right) \mid \leqslant c r_{3}^{-(N-(i+1))}+c h \leqslant c r_{1} r_{1}^{-(N-i)}+c h .
$$

Hence, we obtain from Lemmas 3.2, 4.2, and 3.1,

$$
\left|z\left(x_{i}\right)-z_{h i}^{1}\right| \leqslant c h r_{1}^{-(N-(i+1))}+c h \leqslant c h .
$$

It remains to bound the $v$ term on the right side of (4.4) in the case $h \geqslant \epsilon$. From the definition of $v(x),|L v(x)| \leqslant c \epsilon^{-1} v(x)$. Since $v\left(x_{i}\right) \leqslant r_{3}^{-(N-i)}$, we have

$$
\left|L_{h}^{1} v_{h i}^{1}\right|=\left|L v\left(x_{i}\right)\right| \leqslant c \epsilon^{-1} r_{3}^{-(N-i)} \leqslant c h^{-1} r_{1}^{-(N-i)},
$$

where we have used Lemma 4.1(a) with $k=1$. Hence, from Lemmas 4.2 and 3.1,

$$
\left|v_{h i}^{1}\right| \leqslant c r_{1}^{-(N-i)}
$$

so

$$
\left|v\left(x_{i}\right)-v_{h i}^{1}\right| \leqslant\left|v\left(x_{i}\right)\right|+\left|v_{h i}^{1}\right| \leqslant c r_{1}^{-(N-i)}, \quad 1 \leqslant i \leqslant N-1 .
$$

The desired estimate then follows from Lemma 4.1(b), and the proof is complete.

We next have, for the operator $L_{h}^{2}$,

THEOREM 4.2. There is a constant $c>0$, independent of $h$ and $\epsilon$, such that

$$
\begin{aligned}
& \left|y\left(x_{i}\right)-y_{h i}^{2}\right| \leqslant \frac{c h^{2}}{h+\epsilon}\left[1+\epsilon^{-1} \exp \left(-\bar{a} \epsilon^{-1}\left(1-x_{i}\right)\right)\right], \quad h \leqslant \epsilon, \\
& \left|y\left(x_{i}\right)-y_{h i}^{2}\right| \leqslant c\left[\frac{h^{2}}{h+\epsilon}+\exp \left(-a\left(1-x_{i}\right) /(a h+\epsilon)\right)\right], \quad h \geqslant \epsilon,
\end{aligned}
$$

where $\bar{a}$ is as in Lemma 4.1 .

Proof. The proof is the same as that of Theorem 4.1, except that (3.2) is used instead of (3.1) to estimate the truncation error, and $r_{2}$ is used in place of $r_{1}$.

To analyse the Il'in scheme, we shall use the decomposition $y=\gamma v+z$ both when $h \leqslant \epsilon$ and when $h \geqslant \epsilon$. In the next lemma, we give a bound for $v-v_{h}^{3}$. Note that if $p(x)$ is a constant, then $v_{h}=v$ and the lemma is not needed.

LEMMA 4.3. $\left|v\left(x_{i}\right)-v_{h i}\right| \leqslant c h^{2} /(h+\epsilon)$, where $c>0$ is independent of $\epsilon$. 
Proof. A computation gives

$$
\begin{aligned}
L v(x) & =-\epsilon^{-1} p(1)[p(1)-p(x)] v(x)+q(x) v(x) \\
L_{h}^{3} v & =-\frac{2 p(x) \sinh \left(1 / 2 p(1) h \epsilon^{-1}\right) \sinh \left(1 / 2 h \epsilon^{-1}[p(1)-p(x)]\right)}{h \sinh \left(1 / 2 p(x) h \epsilon^{-1}\right)} v(x)+q(x) v(x)
\end{aligned}
$$

We use the approximation $\sinh \xi=\xi+S$, where $|S| \leqslant c|\xi|^{3}\left(1+\xi^{2}\right)^{-1} e^{|\xi|}$. We have

$$
\begin{aligned}
\tau(x)= & L v(x)-L_{h}^{3} v(x) \\
= & 2 p(x) \frac{\left[1 / 2 p(1) h \epsilon^{-1}+S_{1}\right]\left[1 / 2 h \epsilon^{-1}(p(1)-p(x))+S_{2}\right]}{1 / 2 h^{2} p(x) \epsilon^{-1}+h S_{3}} v(x) \\
& -\epsilon^{-1} p(1)[p(1)-p(x)] v(x) \\
\tau(x)= & \left\{p(x) h \epsilon^{-1}[p(1)-p(x)] S_{1}\right. \\
& +p(x) p(1) h \epsilon^{-1} S_{2}+2 p(x) S_{1} S_{2} \\
& \left.\cdot-h \epsilon^{-1} p(1)[p(1)-p(x)] S_{3}\right\} v(x) / h \sinh \left(1 / 2 p(x) h \epsilon^{-1}\right)
\end{aligned}
$$

where

$$
\begin{aligned}
& \left|S_{1}\right| \leqslant \frac{c h^{3}}{\epsilon\left(h^{2}+\epsilon^{2}\right)} \exp \left(1 / 2 p(1) h \epsilon^{-1}\right) \\
& \left|S_{2}\right| \leqslant \frac{c h^{3}(1-x)}{\epsilon(h+\epsilon)^{2}} \exp \left(c(1-x) h \epsilon^{-1}\right) \\
& \left|S_{3}\right| \leqslant \frac{c h^{3}}{\epsilon\left(h^{2}+\epsilon^{2}\right)} \exp \left(1 / 2 p(x) h \epsilon^{-1}\right)
\end{aligned}
$$

and where we have used the inequality $|p(1)-p(x)| \leqslant c(1-x)$. Using the inequality $\sinh \xi \geqslant c \xi(1+\xi)^{-1} e^{\xi}, \xi>0$, we see that the denominator in (4.5) is bounded from below by $c h^{2}(h+\epsilon)^{-1} \exp \left(1 / 2 h \epsilon^{-1} p(x)\right)$. The numerator in (4.5) consists of four terms. We bound each of these terms as follows:

$$
\begin{gathered}
\left|p(x) h \epsilon^{-1}[p(1)-p(x)] S_{1}\right| \leqslant c h \epsilon^{-1}(1-x) \cdot h^{3} \epsilon^{-1}\left(h^{2}+\epsilon^{2}\right)^{-1} \exp \left(1 / 2 p(1) h \epsilon^{-1}\right) \\
\leqslant c h^{4} \epsilon^{-2}(1-x)(h+\epsilon)^{-2} \exp \left(1 / 2 p(1) h \epsilon^{-1}\right) ; \\
\left|p(x) p(1) h \epsilon^{-1} S_{2}\right| \leqslant c h^{4} \epsilon^{-2}(1-x)(h+\epsilon)^{-2} \exp \left(c(1-x) h \epsilon^{-1}\right) ; \\
\left|2 p(x) S_{1} S_{2}\right| \leqslant c h^{4} \epsilon^{-2}(1-x)(h+\epsilon)^{-2} \exp \left[1 / 2 p(1) h \epsilon^{-1}+c(1-x) h \epsilon^{-1}\right] \\
\left|h \epsilon^{-1} p(1)[p(1)-p(x)] S_{3}\right| \leqslant c h^{4} \epsilon^{-2}(1-x)\left(h^{2}+\epsilon^{2}\right)^{-1} \exp \left(1 / 2 p(x) h \epsilon^{-1}\right) .
\end{gathered}
$$

Using these inequalities in (4.5), we obtain

$$
|\tau(x)| \leqslant \frac{c h^{2}(1-x)}{\epsilon^{2}(h+\epsilon)} \exp \left(c(1-x) h \epsilon^{-1}\right) \nu(x) .
$$

Let $b=1 / 2(p(1)-a)$, so $b>0$. We may find a constant $c_{1}>0$ so that when $h \leqslant$ 
$c_{1}, p(1)-a-c h \geqslant b$. Then we have, for $h \leqslant c_{1}$,

$$
\begin{aligned}
& |\tau(x)| \leqslant \frac{c h^{2}(1-x)}{\epsilon^{2}(h+\epsilon)} \exp \left(-a \epsilon^{-1}(1-x)\right) \exp \left(-b \epsilon^{-1}(1-x)\right) \\
& |\tau(x)| \leqslant \frac{c h^{2}}{\epsilon(h+\epsilon)} \exp \left(-a \epsilon^{-1}(1-x)\right)
\end{aligned}
$$

At the mesh point $x_{i},(4.6)$ yields

$$
\left|\tau\left(x_{i}\right)\right|=\left|L_{h}^{3}\left(v_{i}-v\left(x_{i}\right)\right)\right| \leqslant \frac{c h^{2}}{\epsilon(h+\epsilon)} r_{3}^{-(N-i)} .
$$

We now use Lemma 4.2 and Lemma 3.1 to obtain a bound for $v_{i}-v\left(x_{i}\right)$. If $h \leqslant \epsilon$, $h \leqslant c_{1}$, we have

$$
\left|v\left(x_{i}\right)-v_{i}\right| \leqslant \frac{c h^{2}}{h+\epsilon} r_{3}^{-(N-i)} \leqslant \frac{c h^{2}}{h+\epsilon} .
$$

If $h \geqslant \epsilon, h \leqslant c_{1}$, we have

$$
\left|v\left(x_{i}\right)-v_{i}\right| \leqslant \frac{c h^{2}}{h+\epsilon} \cdot h \epsilon^{-1} r_{3}^{-(N-i)}
$$

Since $i \leqslant N-1,1-x_{i} \geqslant h$, and

$$
h \epsilon^{-1} r_{3}^{-(N-i)}=h \epsilon^{-1} \exp \left(-a \epsilon^{-1}(1-x)\right) \leqslant h \epsilon^{-1} \exp \left(-a h \epsilon^{-1}\right) \leqslant c,
$$

so the inequality is obtained in this case. There remains the case $h \geqslant c_{1}$. For this, it suffices to show that $v$ and $v_{h}$ are bounded for all $h \geqslant c_{1}, \epsilon \leqslant 1$. This is true by inspection for $v$. To bound $v_{h}$, we note that $L_{h}^{3} v_{h}=L v$ is bounded for all $h$ and $\epsilon$. Hence, from Lemmas 3.1 and $3.2, v_{h}$ is bounded for all $h$ and $\epsilon$. This completes the proof of the lemma.

We shall give two error bounds for the Il'in scheme. Our first bound was also given in [2].

THEOREM 4.3. There is a constant $c>0$, independent of $h$ and $\epsilon$, such that $\left|y\left(x_{i}\right)-y_{h i}^{3}\right| \leqslant c h$.

Proof. Using the decomposition of Lemma 2.4, let $\tau_{i}=L_{h}^{3}\left(z-z_{h}\right)=L_{h}^{3} z-$ Lz. Using (3.1) with $k=3$, and (2.7),

$$
\begin{aligned}
\left|\tau_{i}\right| & \leqslant c \int_{x_{i-1}}^{x_{i+1}}\left[\epsilon\left|z^{(3)}(t)\right|+\left|z^{(2)}(t)\right|\right] d t \\
& \leqslant c h+c \epsilon^{-1} \int_{x_{i-1}}^{x_{i+1}} \exp \left(-a \epsilon^{-1}(1-t)\right) d t \\
& \leqslant c h+c \sinh a h \epsilon^{-1} \cdot \exp \left(-a \epsilon^{-1}\left(1-x_{i}\right)\right) \\
& =c h+c \sinh a h \epsilon^{-1} \cdot r_{3}^{-(N-i)} .
\end{aligned}
$$

Using Lemmas $3.2,4.2$, and 3.1 , we obtain 


$$
\begin{aligned}
\left|z\left(x_{i}\right)-z_{h i}\right| & \leqslant c h+c \max (h, \epsilon) \sinh a h \epsilon^{-1} \cdot \exp \left(-a \epsilon^{-1}\left(1-x_{i}\right)\right) \\
& \leqslant c h+c \max (h, \epsilon)\left[1-\exp \left(-2 a h \epsilon^{-1}\right)\right]
\end{aligned}
$$

For $h \leqslant \epsilon$, using the inequality $1-e^{-t} \leqslant c t, t>0$, we get $\left|z\left(x_{i}\right)-z_{h i}\right| \leqslant c h$. For $h \geqslant \epsilon$, we also obtain this inequality. Hence, using Lemma 4.3 and the triangle inequality, we obtain the result.

THEOREM 4.4. There is a constant $c>0$, independent of $h$ and $\epsilon$, such that

$$
\left|y\left(x_{i}\right)-y_{h i}^{3}\right| \leqslant \frac{c h^{2}}{h+\epsilon}+\frac{c h^{2}}{\epsilon} \exp \left(-a \epsilon^{-1}\left(1-x_{i}\right)\right) .
$$

Proof. Again setting $\tau_{i}=L_{h}^{3}\left(z-z_{h}\right)$, we have from (3.3) and (2.7),

$$
\begin{aligned}
\left|\tau_{i}\right| \leqslant & c \int_{x_{i-1}}^{x_{i+1}}\left[h \epsilon\left|z^{(4)}\right|+h\left|z^{(3)}\right|+\frac{h}{h+\epsilon}\left|z^{(2)}\right|\right] d t \\
\leqslant & \frac{c h^{2}}{h+\epsilon}+\frac{c h}{h+\epsilon} \sinh a h \epsilon^{-1} \cdot \exp \left(-a \epsilon^{-1}\left(1-x_{i}\right)\right) \\
& +c h \epsilon^{-1} \sinh a h \epsilon^{-1} \exp \left(-a \epsilon^{-1}\left(1-x_{i}\right)\right) \\
\leqslant & c h^{2}(h+\epsilon)^{-1}+c h \epsilon^{-1} \sinh a h \epsilon^{-1} \cdot r_{3}^{-(N-i)} .
\end{aligned}
$$

Using Lemmas 3.2, 4.2, and 3.1, we obtain

$$
\left|z\left(x_{i}\right)-z_{h i}\right| \leqslant \frac{c h^{2}}{h+\epsilon}+c h \epsilon^{-1} \max (h, \epsilon) \sinh a h \epsilon^{-1} \cdot \exp \left(-a \epsilon^{-1}\left(1-x_{i}\right)\right)
$$

For $h \leqslant \epsilon$, we use the inequality $\sinh a t \leqslant c t, t \leqslant a$, to get

$$
\left|z\left(x_{i}\right)-z_{h i}\right| \leqslant \frac{c h^{2}}{h+\epsilon}+c h^{2} \epsilon^{-1} \exp \left(-a \epsilon^{-1}\left(1-x_{i}\right)\right)
$$

Using the triangle inequality and Lemma 4.3, we obtain the bound for the error in this case. For $h \geqslant \epsilon, h \leqslant 2 h^{2}(h+\epsilon)^{-1}$, and the result follows from Theorem 4.3.

The difference operators $L_{h}^{2}$ and $L_{h}^{3}$ have, for $\epsilon>0$, a truncation error that is $O\left(h^{2}\right)$, whereas the reduced difference operators, obtained by letting $\epsilon \rightarrow 0$, have a truncation error that is $O(h)$. We shall now show that this loss of an order of accuracy near $\epsilon=0$ holds for all tridiagonal difference operators of positive type. For this, it suffices to consider the case of constant coefficients, $p(x) \equiv p>0, q(x) \equiv$ $q \geqslant 0$. We consider the difference operator

$$
\left(L_{h} u\right)_{i}=r(h, \epsilon) u_{i+1}+s(h, \epsilon) u_{i}+t(h, \epsilon) u_{i-1} .
$$

We suppose that $r, s$, and $t$ are continuous functions of $(h, \epsilon)$ for $h>0, \epsilon \leqslant 1$. We say that the difference operator is of positive type if $s(h, \epsilon)>0, r(h, \epsilon)<0, t(h, \epsilon)$ $<0$. If $y(x)$ is a smooth function, we denote the truncation error by

$$
\begin{aligned}
\tau(x, h, \epsilon)= & r(h, \epsilon) y(x+h)+s(h, \epsilon) y(x)+t(h, \epsilon) y(x-h) \\
& +\epsilon y^{(2)}(x)-p y^{(1)}(x)-q y(x) .
\end{aligned}
$$


With these notations we have

THEOREM 4.5. Suppose that, for any smooth function $y(x)$, the truncation error satisfies $|\tau(x, h, \epsilon)| \leqslant c h^{\delta}$ where $\delta>1$ and where $c$ does not depend on $x, h$, or $\epsilon$, then the difference operator is not of positive type for all $\epsilon$ and $h$ sufficiently small.

Proof. Letting $y$ be a quadratic polynomial, calculating $\tau(x)$, and comparing coefficients, we obtain

$$
\begin{aligned}
& r+s+t=q+\mu(x, h, \epsilon), \\
& h(r-t)=p+\lambda(x, h, \epsilon), \\
& 1 / 2 h^{2}(r+t)=-\epsilon+\eta(x, h, \epsilon),
\end{aligned}
$$

where $|\eta|,|\lambda|,|\mu| \leqslant c h^{\delta}$, uniformly in $(x, h, \epsilon)$. Solving this system of equations, we obtain

$$
r(h, \epsilon)=-\frac{\epsilon}{h^{2}}+\frac{p}{2 h}+\frac{\eta(x, h, \epsilon)}{h^{2}}+\frac{\lambda(x, h, \epsilon)}{2 h} .
$$

Then $r(h, 0)=p / 2 h+O\left(h^{-1+\delta}\right)$, so for $h$ sufficiently small, $r(h, 0)>0$. Hence, for each $h>0$ sufficiently small, there is an $\epsilon>0$ sufficiently small, such that for this $h$ and $\epsilon$, the difference approximation is not of positive type.

5. Numerical Results. We give some numerical results for our difference schemes, as applied to the problem $-\epsilon y^{\prime \prime}+y^{\prime}=1$ with boundary conditions $y(0)=$ $0, y(1)=0$. In addition to using the difference operators $L_{h}^{1}$ and $L_{h}^{2}$ we have used the centered difference operator

$$
L_{h}^{0} u_{i}=-D_{+} D_{-} u_{i}+D_{0} u_{i}, \quad D_{0} u_{i}=\left(u_{i+1}-u_{i-1}\right) / 2 h .
$$

This difference operator gives an $O\left(h^{2}\right)$ approximation to the differential equation, but is known to give poor results for small $\epsilon$ [1]. The three figures give results of computations using $h=0.02$ and for three different values of $\epsilon$. In Figures 1 and 2 we have plotted the errors in the approximate solutions. Denoting the errors in using $L_{h}^{k}$ by $e^{k}, e^{0}, e^{1}$, and $e^{2}$ are represented respectively by the solid line, the dashed line, and the long dashed line. For $\epsilon=0.1$, Figure 1 shows that $L_{h}^{2}$ produces a solution that is almost as accurate as that produced by $L_{h}^{0}$, while the first order scheme gives a much larger error. For $\epsilon=0.01<h$, Figure 2 shows that $e^{2}(x)$ is smaller than $e^{0}(x)$, and $e^{1}(x)$ is the largest error. This indicates that $L_{h}^{2}$ gives the most accurate solution. In Figure 3 we present results for $\epsilon=0.001 . e^{2}(x)$ is very close to zero in the entire interval, and $e^{1}(x)$ is close to zero except near $x=1$. The oscillating solid line is the centered difference solution $u^{0}(x)$ which we have shown superimposed on the true solution, $y(x)$. This figure indicates that the use of $L_{h}^{2}$ gives a very good approximation, while the centered scheme is worthless. For this problem, the scheme $L_{h}^{3}$ gives the exact answer. In conclusion, the difference operators $L_{h}^{2}$ and $L_{h}^{3}$ provide accurate solutions to a singular perturbation problem without turning points over a wide range of values of $h$ and $\epsilon$. 


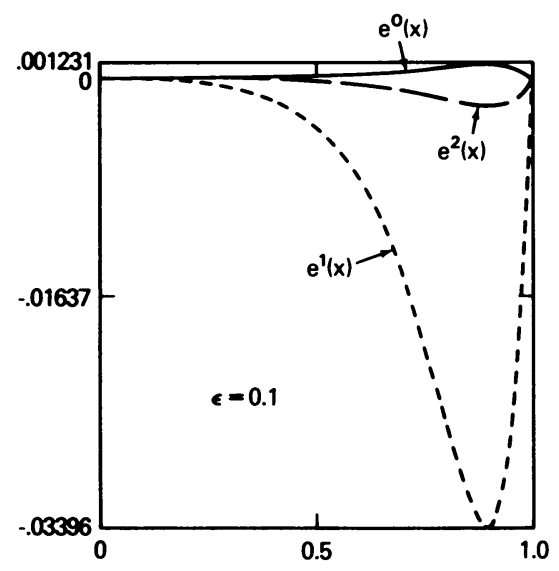

Figure 1

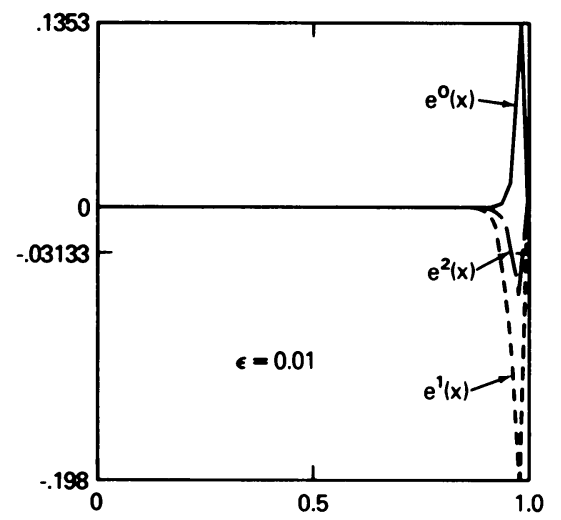

Figure 2

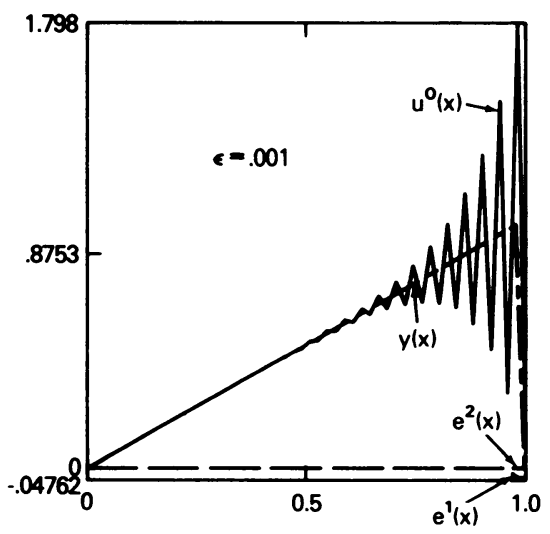

FIGURE 3 
Acknowledgement. The authors thank Dr. Alan Berger for many helpful comments.

Institute of Physical Science and Technology

University of Maryland

College Park, Maryland 20742

Section on Theoretical Biophysics

National Heart, Lung and Blood Institute, and

Mathematical Research Branch

National Institute of Arthritis, Metabolism, and Digestive Deseases

National Institutes of Health

Bethesda, Maryland 20014

1. F. W. DORR, "The numerical solution of singular perturbations of boundary value problems," SIAM J. Numer. Anal., v. 7, 1970, pp. 281-313.

2. A. M. IL'IN, "Differencing scheme for a differential equation with a small parameter affecting the highest derivative," Mat. Zametki, v. 6, 1969, pp. 237-248= Math. Notes, v. 6, 1969 , pp. 596-602.

3. K. E. BARRETT, "The numerical solution of singular-perturbation boundary-value problems," J. Mech. Appl. Math., v. 27, 1974, pp. 57-68.

4. L. R. ABRAHAMSSON, H. B. KELLER \& H. O. KREISS, "Difference approximations for singular perturbations of systems of ordinary differential equations," Numer. Math., v. 22, 1974, pp. 367-391.

5. J. E. FLAHERTY \& R. E. O'MALLEY, JR., "The numerical solution of boundary value problems for stiff differential equations," Math. Comp., v. 31, 1977, pp. 66-93.

6. M. H. PROTTER \& H. F. WEINBERGER, Maximum Principles In Differential Equations, Prentice-Hall, Englewood Cliffs, N. J., 1967.

7. R. S. VARGA, Matrix Iterative Analysis, Prentice-Hall, Englewood Cliffs, N. J., 1962.

8. P. J. DAVIS, Interpolation and Approximation, Blaisdell, Waltham, Mass., 1963.

9. V. A. GUSHCHIN \& V. V. SHCHENNIKOV, "A monotonic difference scheme of secondorder accuracy," U.S.S.R. Computational Math. and Math. Phys., v. 14, 1974, pp. 252-256. 